\title{
LA PLASMACIÓN DEL ARTE Y DE LA CULTURA GRECOLATINA A TRAVÉS DE UNA VISIÓN JAPONESA LOS CABALLEROS DEL ZODÍACO (SAINT SEIYA) Y SAILOR MOON
}

\author{
THE PLASMATION OF ART AND GRECOLATINE \\ CULTURE THROUGH A JAPANESE VISION \\ THE KNIGHTS OF THE ZODIAC (SAINT SEIYA) AND \\ SAILOR MOON
}

\author{
Carmen María Bersabé Cabezuelo \\ (Historia del Arte, Universidad de Valencia, España) \\ carberca@alumni.uv.es
}

Recibido: 15 febrero 2020 Aceptado: 07 marzo 2020

\begin{abstract}
Resumen: Análisis de los elementos mitológicos y artísticos grecolatinos en las series Saint Seiya (Los Caballeros del Zodíaco) de Masami Kurumada (1985) y Sailor Moon de Naoko Takeuchi (1992).
\end{abstract}

Palabras clave: Arte; grecolatina; animación; Sailor Moon, Saint Seiya.

\begin{abstract}
Analysis of the most outstanding Greco-Latin mythological and artistic elements in Saint Seiya (The Knights of the Zodiac) by Masami Kurumada (1985) and Sailor Moon by Naoko Takeuchi (1992).
\end{abstract}

Keywords: Art; greco-Latin; animation; Sailor Moon; Saint Seiya.

\section{INTRODUCCIÓN}

Tanto el cómic (manga) como la animación (anime) japonesa hace ya varias décadas que tienen un puesto preeminente fuera de las fronteras del país nipón, dando como resultado uno de los mercados más importantes junto al americano y el europeo.

Este escenario, en numerosas ocasiones da lugar a que se establezca un vínculo entre dos núcleos culturales tan diferentes como lo son el occidental y el oriental; lo que 
a su vez lleva a crear una corriente de influencias que funciona en ambas direcciones. La relación instituida da como lugar obras que hibridan características de ambos como por ejemplo en el caso que nos ocupa: Saint Seiya y Sailor Moon (Navok, 2009, pp.21-30).

Estas dos series establecen como una de su más importante fuente de información e inspiración el imaginario de la cultura grecolatina; aunque si bien es cierto que existen referencias a otras mitologías en las cuales no ahondaremos. Es fundamental la representación artística que de estos mitos se hace a lo largo de la historia, y que de una manera reimaginada y ficcionada, aparece en las series antes mencionadas.

Ambas narraciones retoman autores clásicos tales como Ovidio, especialmente "Las metamorfosis" (Ovidio, 2007, pp.150-153), no obstante, no es el único referente; y se unen al manga shonen y el shojo.

El manga shonen ("chico") teóricamente orientado a un público mayoritariamente masculino de entre 12 a 18 años, englobando obras como Saint Seiya o Dragon Ball (Toriyama, 1984). Estas publicaciones tienen características comunes: un protagonista, algo torpe y sin potencial que lo haga destacar de entre el grupo de jóvenes que se ven envueltos en un forzoso viaje con intención de encontrar un determinado ítem en el cual irán conociendo el mundo que les rodea; descubriendo que puede llegar a ser alguien a destacar. A este protagonista (masculino, dato a recordar) se le unen una serie de aliados, que, con sus personalidades, además de sus poderes, le ayudan y complementan. Unido a ello, no debemos olvidar la figura del maestro que le guíe, muchas veces marcado por las palabras antaño dictadas por una profecía y que dirime la serie en distintos caminos.

Este camino, lleno de incertidumbre, luchas, descubrimientos tanto propio como ajeno sigue lo establecido por Joseph Campbell en "El héroe de las mil caras", obra en la cual desgrana los pasos más repetidos por el héroe a la hora de iniciar su periplo (Campbell, 2014, pp.151-153) y las situaciones peligrosas que le acechan en el camino para regresar al hogar.

Dentro del manga shonen, existen muchas variaciones, pero la más extendida es la de una serie sobre peleas en la que el más poderoso es quién tiene derecho a sobrevivir 
y proseguir su metafórico viaje, casos como Ring ni Kakero, de Masami Kurumada, también autor de Saint Seiya; lo mismo que sucede en Bleach, Naruto o Dragon Ball, entre otros.

Por otro lado, también existen creaciones que se enmarcan demográficamente dentro del shonen: son las basadas en el mundo de los deportes. Estos productos no abandonan la lucha ni la intención de conseguir un objetivo, pero que a través del esfuerzo (el camino del héroe), los compañeros y los entrenadores (maestro) parece al alcance de la mano. Series como Slam Dunk (Inoue, 1990) sobre el mundo del baloncesto estudiantil, o Haikyū (Furudate, 2012) acerca del voleibol estudiantil dan buena muestra de ello.

El shonen es capaz de abarcar y unir lo más diverso dando como resultado un producto atractivo y apasionante, sucediendo lo mismo con su contraparte femenina en lo que a manga se refiere: el género shojo.

La definición de shojo ("chica joven"), al igual que la de shonen, abarca un determinado grupo poblacional; el de aquellas muchachas que están cerca de convertirse en mujeres (entre 10 y 18 años). Al tratarse de un conjunto de obras enfocadas a una mayoría femenina, una de las características principales es el enfoque romántico tradicional de dichas obras. Esta denominación es bastante más flexible en la actualidad y el romance puede no ser lo principal en estas historias. Al igual que sucede con su género hermano el shonen, el shojo abarca una inmensidad de subgéneros.

El shojo primigenio surge, entre otros autores y autoras, de la mano del padre del manga Osamu Tezuka y su obra "La Princesa Caballero" (1967), en la cual la joven princesa es hecha pasar por príncipe desde su más tierna infancia hasta que el amor llega a su vida. Esta no es la única obra en la cual la princesa ha de hacerse pasar por hombre debido a las circunstancias como sucede en la famosa "La Rosa de Versailles" (Ikeda, 1972); manga de carácter histórico que se desarrolla en la Francia de María Antonieta y donde, la protagonista, Oscar François de Jarjayes, es criada como hombre para así poder ser la heredera de su padre (Martínez, 2016, p.31). 
Caben destacar las siguientes divisiones que se dan en el shojo: el romance adolescente (de variable cómica o dramática) que se da dentro del colegio/ instituto, donde la protagonista va evolucionando con ayuda de nuevas amistades (similar al viaje del héroe de Campbell) y en las que habitualmente conoce al arquetípico protagonista masculino por el cual se siente atraída, incluso con sus diferencias. Obras de corte costumbrista, en las cuales el romance es dirigido por las circunstancias de la vida y que puede gustar a un público más adulto como Maison Ikkoku (1980-1987) de Rumiko Takahashi, una de las autoras más importantes del shojo y del género fantástico.

Sin embargo, uno de los subgéneros más importantes que ha ido adquiriendo una entidad propia es el de las magical girls. Como podemos deducir de su designación, se trata de chicas con poderes mágicos, chicas que definitivamente sí van a encaminarse al sendero que marca como cierto la obra de Campbell, "El héroe de las Mil Caras". Una senda marcada por la amistad, la magia, el encuentro con la figura del maestro, con los personajes adorables y con los objetos portadores, conductores y potenciadores del poder latente de estas jóvenes.

El inicio de las magical girls lo encontramos con el arriba mencionado "La Princesa Caballero" y continuará con "Sally La Bruja" (1966). Esto solo será el comienzo y en años próximos irá evolucionado lentamente hasta llegar a la que hemos seleccionado, Sailor Moon (Takeuchi, 1992), exquisita muestra del shojo de magical girls, que va un paso más allá y nos muestra cómo con el poder de la amistad una mujer es capaz de salvarse a sí misma sin necesidad de convertirse en una damisela en apuros (Napier, 2005, p.33).

En las historias seleccionadas, Saint Seiya y Sailor Moon, podemos rastrear la presencia clásica, ejemplifican la influencia de una tradición antigua como la grecolatina en unas series del país nipón y que no son, ni mucho menos el único ejemplo. Toman aquello que les resulta apasionante y lo magnifican, dando como resultado dos de las series más destacadas de la historia de la animación y del cómic japonés contemporáneo, con capacidad para atraer a público de todas las edades y de todos los países, siendo sagas longevas y muy estimadas tanto por el lector japonés como por el occidental. 


\section{EL VALOR DE SAILOR MOON Y SAINT SEIYA}

En las historias seleccionadas, Saint Seiya y Sailor Moon, podemos rastrear la presencia clásica, ejemplifican la influencia de una tradición antigua como la grecolatina en unas series del país nipón y que no son, ni mucho menos el único ejemplo. Toman aquello que les resulta apasionante y lo magnifican, dando como resultado dos de las series más destacadas de la historia de la animación y del comic japonés contemporáneo, con capacidad para atraer a público de todas las edades y de todos los países, siendo sagas longevas y muy estimadas tanto por el lector japonés como por el occidental.

Tanto Masami Kurumada (Saint Seiya) como Naoko Takeuchi (Sailor Moon), hicieron una exhaustiva investigación sobre la cultura grecolatina tanto su mitología como su arquitectura para ser lo más fieles posibles o en el caso de modificar su base, que toda la estructura resultante siguiera teniendo lógica narrativa. Para ello, Masami Kurumada realizaría varios viajes a Grecia para tomar referencias, especialmente en el recinto arqueológico de Atenas; complejo que aparece numerosas veces en Saint Seiya como veremos en las menciones arquitectónicas. Su pasión por la cultura clásica llega hasta tal punto que toda su investigación no cabe en una única obra y habrá de abarcar una serie de precuelas/secuelas que completan la publicación original.

Naoko Takeuchi, sin embargo, no llegaría a realizar viaje alguno a Grecia, pero si llevó a cabo una gran investigación, hecho que queda patente en los personajes de Sailor Moon y algunas de las referencias internas como el hecho de que la protagonista, Usagi/ Selene (la diosa lunar previa a Artemisa) tenga en su pareja al príncipe de la tierra, Mamoru/ Endimión.

Ambos relatos, crean una nueva mitología propia basada en lo que denominamos clásico. Para ello, inician su relato en un mundo contemporáneo (1980 en el caso de Saint Seiya, 1990 en el de Sailor Moon) ajenos al futuro que les aguarda. Unas vidas, cuyo destino ya estaba regido por la fuerza de las estrellas y sus constelaciones: en el caso de Saint Seiya, los caballeros o Saints, están bajo la advocación de una determinada constelación lo que nos lleva a la denominación de la serie en castellano. El cambio se debe esencialmente a las connotaciones católicas asociadas al término Saint (traducido 
literalmente por Santo) y que hizo que numerosos grupos cristianos exigieran el cambio por entenderlo como un ataque a sus creencias. Los caballeros, conforman una orden militar al servicio de la protección exclusiva de la diosa Atenea, que se reencarna cada 200 años en su continuo intento de frenar la codicia de Hades respecto a la tierra. Para ello, los caballeros se dividen en tres niveles: bronce (nivel inferior), plata (nivel intermedio, suelen ser los maestros de los caballeros de bronce) y oro, el nivel superior y en las manos de los cuales descansa la protección directa de la diosa; puesto que custodian los templos (también llamados casas) que llevan hasta las entrañas más profundas del santuario, lugar en que se refugia Atenea. Lo llamativo de la estructura de los caballeros, es que responde a las edades mitológicas del hombre (Ovidio, 2007, pp-70-72), siendo los humanos primigenios, de mayor calidad moral sin problemas ni miserias los primeros que surgieron (oro); seguidos de la edad de plata, mucho más violentos y terminando con la edad de bronce, que, con asombro por parte de los dioses, dio lugar a una generación de personas virtuosas y justas (Hesíodo, Trabajos y días, pp.63-66).

De este modo se traza la organización del santuario, siguiendo sus constelaciones, mientras que en Sailor Moon, los personajes se encuentran bajo el influjo del poder de los planetas del sistema solar y de los dioses a ellos asociados. Con lo expuesto hasta el momento, observamos que existen nutridos ejemplos de la presencia mitológica grecorromana en ambas series.

\section{LA IMPORTANCIA DE LA LUNA Y EL SOL EN SAILOR MOON Y SAINT SEIYA: SELENE, ARTEMISA, APOLO Y HELIOS}

Esta importancia radica en el uso de tres figuras fundamentales y una cuarta en menor medida: la diosa lunar Selene, la diosa lunar Artemisa, el dios solar Helios y la breve aparición del dios solar Apolo, hermano gemelo de Artemisa.

En el caso de Selene (Elvira Barba, 2008, pp.195-196), su presencia es fundamental en Sailor Moon, puesto que es una de las pasadas y futuras encarnaciones de la protagonista, cuyo nombre, Usagi Tsukino, está vinculado con el conejo, que según la mitología japonesa vive en la luna (Tsuki No Usagi). Selene, diosa griega de la luna, es la personificación primera de ésta. Hermana de Helios (el sol), portaba el carro de la 
noche con sus dos plateados corceles. Según la fuente consultada, la cantidad de amantes e hijos varía, pero el punto en el que coinciden es en que Endimión (Graves, 2011, pp.310312) es su pareja más reconocida y, por tanto, la más representada en el arte. En el relato mitológico original es un del cual queda prendada Selene, que baja del cielo cada noche a visitarlo y que tuvo con él 50 hijas. Estas hijas son las llamadas Menae, que representarían cada uno de los meses lunares existentes en un período de cuatro años, de Olimpiada a Olimpiada.

La aparición de Helios (Elvira Barba, 2008, pp.182-184) en Sailor Moon es a través de un Pegaso (Ovidio, 2007, pp.167-168), cuyo resultado da una representación que mezcla su cuerno original con las alas de un unicornio. Este mito establece un punto de conexión con los Campos Elíseos, lugar del cual es sacerdote en su forma humana (Argal Sotés, 2011, pp.80-81). En la serie, sirve como contraparte solar a Chibiusa (Pequeña Usagi en japonés, y por extensión, pequeña luna o pequeño conejo lunar).

Mitológicamente hablando, Helios es el hermano de Selene y, a su vez, personificación del sol y primer dios de éste. Al igual que ella, era portador de un carro, el solar.

La presencia de la diosa lunar Artemisa y del dios solar Apolo, es bastante breve en el universo de Saint Seiya puesto que aparecen únicamente en la película "Saint Seiya. Tenkai Hen: Overture" (Martínez, 2016, pp.134-136). Artemisa es la sustituta de la diosa lunar Selene, aunque sus potestades están muy ligadas al mundo de la caza, también al de la virginidad. Es por ello, que sus atributos principales en su representación artística son el arco (arma que aparece en la película de Saint Seiya) y los ciervos. Tratándose de la gemela de Apolo, se entiende el hecho de que sea este dios quien sustituyera a Helios en su papel de dios solar (Elvira Barba, 2008, pp.185-195). Como mencionamos al principio de este apartado, Apolo es la aparición de menor importancia en la dualidad lunar- solar que establecen las dos sagas; puesto que su aparición iniciaba una trilogía de películas que culminaría con la aparición de Zeus y que no se llevaron a cabo finalmente. 


\section{TRANSFORMACIÓN: LOS DIOSES CLÁSICOS SON MUJERES GUERRERAS.}

Siguiendo esta línea de investigación, está el uso referido a las personalidades y a los atributos vinculados tradicionalmente a la diosa y los dioses que dan su nombre a los planetas de nuestro sistema solar. Existe, sin embargo, una importante transformación respecto a las representaciones tradicionales y es que, en este caso, todos los dioses que en origen son masculinos, en Sailor Moon, son encarnados por fuertes mujeres guerreras.

En el caso de Sailor Moon se produce una inversión de la identidad sexual de los personajes como estrategia discursiva. Esto se debe al hecho del sector demográfico al que está dedicado la serie: mujeres adolescentes que quieren verse reflejadas en el contenido audiovisual que consumen.

Estas jóvenes vestidas con el uniforme escolar más reconocible, el de marinera (sailor fuku o seifuku), han nacido en la tierra con la misión de proteger a la princesa de la luna, Selene. Para ello, renacieron junto a ésta para evitar el trágico pasado.

Todas visten una versión modificada del uniforme escolar de marinera, con el color usualmente asociado al planeta y cambios específicos que marcan el carácter de la portadora: el furibundo Marte (Ares) con su color rojo, acompañado de sus inseparables Phobos y Deimos (Miedo y Pena); que en el cómic son dos doncellas que se convierten en cuervos (hecho que por otra parte recuerda a los cuervos del germánico Odín, Hugin y Munin, Pensamiento y Memoria) que protegen a su dueña, Rei Hino, la doncella guerrera protegida por el símbolo de Marte, dios de la guerra. Su fuerte carácter es compartido por Sailor Mars, una miiko, sacerdotisa de la religión shinto (Ono, 2017, pp.65-66) con poderes adivinatorios (a través del fuego) y piroquinesis; vestida de blanco y rojo. Al igual que el planeta que la acoge, está asociada al color rojo y al fuego, aunque a diferencia del dios, no viste con armadura y yelmo.

En contraposición a la colérica (pero de buen corazón) Marte, se encuentra Mercurio (Hermes) azulada entidad, que respeta la esencia del dios que le da nombre y en cuya figura femenina, encontramos una sabiduría difícil de no ver. Sailor Mercury o 
Ami Mizuno es la alumna perfecta del instituto, que sueña con ser médico. De temperamento calmado, sus poderes se asocian al elemento acuático. Uno de sus ataques, Mercury Aqua Rhapsody (en inglés en todas las ediciones) que no es si no, una lira formada por corrientes marinas, lo que nos conecta con el mito de creación de la lira de mano de Mercurio, creada con un caparazón de tortuga y que luego Apolo adquirió para sí. Mercurio, además del dios de la astucia, es el mensajero olímpico, el dios del comercio, incluso de los ladrones.

Júpiter, el equivalente a Zeus tiene en la joven Makoto Kino todo el poder que se le asocia al roble y al rayo, dos de los atributos del padre de todos los dioses olímpicos (menos el águila). De carácter fuerte pero entregada a la gente que le importa, Sailor Jupiter usa su poder para proteger a la princesa Selene de todo mal. Para ello, sus arremetidas contra el enemigo se basan en los atributos antes mencionados, especialmente el roble y el rayo, siendo dos de estos ataques Jupiter Thunder (Trueno) y Jupiter Oak Evolution (Roble) (Argal Sotés, 2011, p.199).

Continuamos con Neptuno, dios del mar (equivalente a Poseidón), y cuyos ataques ahogan al enemigo; establece un fuerte vínculo con Urano. Sailor Neptune/ Michiru Kaioh es una violinista virtuosa que forma parte de las Outer Senshi. Al igual que sucede con Poseidón, usa el tridente como símbolo que aparece en su espejo y que le permite en ocasiones percibir las mareas cambiantes de los sucesos futuros. Su carácter, al igual que sucede con el mar, es variable dependiendo de tu conocimiento sobre ella, que te permite conocerla, navegarla, y en definitiva amarla como hacen los marineros. La presencia del espejo hace referencia asimismo a las tres joyas sagradas de la religión sintoísta, junto con la espada y la gema (Ono, 2017, p.20).

La unión entre Neptuno y Urano es difícil de encontrar en los relatos mitológicos: mientras que Neptuno sería la personificación del mar en su totalidad y rey de todo lo que allí sucede, Urano (Caelus) (Graves, 2011, pp.52-62) sería la personificación del cielo y pareja de la Tierra (Gaia/ Terra); mientras que en Sailor Moon, el vínculo se establece entre Sailor Uranus y Sailor Neptune. Sailor Uranus/ Haruka Tenoh es la portadora de la espada sagrada (joya sagrada sintoísta) con la cual es capaz de rasgar la tierra y el cielo. 
En ella existe una dualidad, creativa y destructiva y llega hasta el género presentándonos tanto en su faceta masculina como en su aspecto femenino.

Plutón/Hades (Graves, 2011, pp.176-183), dios del más allá y del inframundo, es quien guarda la puerta de acceso al cambio, al otro lado, al tiempo que no se puede controlar. Sailor Pluto (Setsuna Meioh), portadora de la llave que contiene el Orbe Granate (la postrera joya sagrada del sintoísmo), tiene la capacidad de abrir ese camino que no debe ser cambiado, la conexión entre el mundo de los vivos y de los muertos. Para atravesar dicho umbral Sailor Pluto se encomienda a Cronos, dios del tiempo (deidad que aparece en la secuela de Saint Seiya, Next Dimension) para que proteja a aquellos que inician allí su peligrosa travesía.

Saturno/Cronos, es quien ciñe la guadaña, el dios durmiente (en Sailor Moon) capaz de acabar con la existencia o reiniciar un mundo nuevo. Esta dualidad, se debe a que, en inicio, Saturno era el dios de la agricultura y con la absorción de otros dioses por parte de los conquistadores, esto cambió (Elvira Barba, 2008, pp.52-57). Sailor Saturn (Hotaru Tomoe) es una joven enfermiza que, bajo el cuidado de su padre, un mad doctor que experimenta con ella, crea un híbrido humano-máquina más propio del ciberpunk.

Por ello, existiendo esa dualidad entre creación y destrucción, en Sailor Moon se teme a Sailor Saturn por no entender que la destrucción que ella pretende es el renacimiento de un mundo sin oscuridad.

No podemos obviar la presencia de la diosa Venus/Afrodita, que conserva su plasmación femenina mientras que Artemis (diosa de la caza) y Luna (equivalente a la personificación de la luna que supone Selene) aparecen bajo una forma gatuna (Parliament, 2014, p.17).

\section{CONCLUSIONES}

Seleccionar estas dos series se debe a criterios tanto estéticos, como históricos y visuales. Hay que mencionar también que no son las únicas series que tratan la mitología dentro 
de sus arcos argumentales, pero si son dos de las que con más profundidad y sobre todo cariño lo han desarrollado.

Tanto Sailor Moon como Saint Seiya han superado ya, en el 2020, su $25^{\circ}$ aniversario en el caso de Sailor Moon y su $30^{\circ}$ en el de Saint Seiya. Cualquier persona podría pensar que esto solo es relevante a nivel de Japón, pero nada más lejos de la realidad, puesto que con motivo de dichos aniversarios se han llevado a cabo nuevos proyectos asociados a las series y muy especialmente a la reedición de estas fuera del país nipón, especialmente en España con una versión nuevamente traducida y de mayor calidad.

La pasión por estas series y por otras de corte similar, no abandonarán a los espectadores mientras exista alguien que a la pregunta ¿Qué te apetece ver hoy? sea capaz de sentir como su cosmos arde o el poder de la luna en su interior.

\section{REFERENCIAS BIBLIOGRÁFICAS}

Argal Sotés, Andrés (2011). Sailor Moon: La Biblia. Manga Books: Mallorca.

- (2017). En nombre de la Luna te castigaré. El Universo mágico de Sailor Moon. Volumen 1. Diábolo Ediciones: Madrid.

- (2018). En nombre de la Luna te castigaré. El Universo mágico de Sailor Moon.

Volumen 2. Diábolo Ediciones: Madrid.

Bernardez, Enrique (2015). Los mitos germánicos. Alianza Editorial: Madrid.

Branca, Roberto (2009). I Cavalieri Dello Zodiaco. Espandi il tuo Cosmo. Iacobelli Editore: Roma.

Cambell, Joseph (2015). El héroe de las mil caras. Psicoanálisis del mito. Fondo de Cultura Económica- Joseph Campbell Foundation: Madrid.

Cartledge, Paul (2004). Los griegos. Encrucijada de la civilización. Critica S.L.: Barcelona.

Elvira Barba, Miguel Ángel (2008). Arte y Mito. Manual de Iconografía Clásica. Sílex Ediciones: Madrid.

Graves, Robert (2011). Los mitos griegos 1. Alianza Editorial: Madrid. 
- (2011). Los mitos griegos 2. Alianza Editorial: Madrid.

Grimal, Pierre (1989). La mitología griega. Editorial Paidós: Barcelona.

- (2010). Diccionario de Mitología Griega y Romana. Editorial Paidós. Madrid.

Hesíodo (2011). Teogonía. Trabajos y días. Alianza Editorial: Madrid.

Mainón, Dominique; Ursini, James. (2006): Modern Amazons: Warrior Women on

Screen. Limelight Editions: Nueva Jersey.

Martínez, Miguel (2009). Los Caballeros del Zodiaco. Un Universo por descubrir.

Dolmen Editorial: Palma de Mallorca.

- (2010). Los Caballeros del Zodiaco. Un Universo por descubrir. Volumen 2.

Dolmen Editorial: Palma de Mallorca.

Martínez, Miguel; Rubio, Néstor. (2013). Los Caballero del Zodiaco. Un universo en Expansión. Dolmen Editorial: Palma de Mallorca.

- (2016). El Infinito cosmos de los caballeros del Zodíaco. ¡Dame tu fuerza, Pegaso! Diábolo Ediciones: Madrid.

Napier, Susan (2005). Anime from Akira to Howl's Moving Castle. Experiencing Contemporary Japanese Animation. Palgrave- Macmillan: Nueva York.

Navok, Jay.; Rudranath, Sushil (2009). Guerreras de leyenda. El reflejo de Japón en Sailor Moon. Ellago Ediciones: Pontevedra.

Ono, Sokyo (2017). Sintoísmo. La vía de los kami. Satori Ediciones: Gijón.

Ovidio (2007). Metamorfosis. Alianza Editorial: Madrid.

Parliament, Roland (2014). Sailor Moon Reflections: The inside story of the original recordings of the English version of Sailor Moon. Friesen Press: Victoria.

Romanello, Elena (2009). Sailor Moon. La bella ragazza guerriera. Iacobelli Editore: Roma.

Tamaki, Saito (2011). Beautiful Fighting Girl. University of Minnesota Press: Minneapolis. 